\title{
Euclidean wormhole solutions of Einstein-Yang-Mills theory in diverse dimensions
}

\author{
Katsuhiko Yoshida and Satoru Hirenzaki \\ Department of Physics, Tokyo Metropolitan University, \\ Setagaya, Tokyo 158, Japan \\ Kiyoshi Shiraishi \\ Institute for Nuclear Study, University of Tokyo, \\ Midori-cho, Tanashi, Tokyo 188, Japan
}

Phys. Rev. D42, 19731981 (1990)

\begin{abstract}
We solve the Euclidean Einstein equations with non-Abelian gauge fields of sufficiently large symmetry in various dimensions. In higherdimensional spaces, we find the solutions which are similar to so-called scalar wormholes. In four-dimensional space-time, we find singular wormhole solutions with infinite Euclidean action. Wormhole solutions in the three-dimensional Einstein-Yang-Mills theory with a Chern-Simons term are also constructed.
\end{abstract}

\section{INTRODUCTION}

Various attempts to quantize gravity have been made by many authors. The path-integral quantization of gravity [1] has been a very popular approach to quantization in the past decade.

A few years ago, a very attractive mechanism to determine the "constants" in nature in the framework of quantum gravity was suggested [2]. The mechanism is based on the appearance of a wormhole configuration in the Euclidean path integral over the distinct topology of spacetime. In particular, the wormhole and baby universes connected by the wormholes have been invoked to explain the vanishing cosmological constants $[2,3]$.

Many problems were pointed out soon after this suggestion, however. For example, the treatment of contributions of large wormholes [4], the efficiency of the dilute gas approximation [5], and the phase of the result of the path integral [6] have been discussed. The validity of Euclidean quantum gravity itself has also been investigated.

Nevertheless we must equip our "theoretical arena" by studying the wormhole solutions to Einstein equations coupled to possible matter fields, until the 
refined formulation of quantum gravity appears. It is suggested that the correct procedure is to sum only over stationary points of the Euclidean action [7]. Thus, we would like to study the various types of wormhole configurations.

We have already known many kinds of wormhole solutions. The following matter fields which support the "throat" of the wormhole were adopted: axion fields [8], scalar fields with and without spontaneous breaking of global $U(1)$ symmetry $[9,10,11,12,13,14,15]$, and $S U(2)$ Yang-Mills fields [16, 17]. Higher-dimensional wormhole solutions were also considered [18], and the higher-derivative correction to the Einstein-Hilbert action was investigated by several authors [19].

Among them, "gauge field wormholes" in Refs. [16] and [17] have very attractive features. Non-Abelian gauge fields are believed to be fundamental entities in unified theories including superstring(-inspired) models [20]. Therefore gauge field wormholes are considered as inevitable objects in the Euclidean formulation. Recently, an interesting class of wormhole solutions has been constructed for the $S U(N)$ Yang-Mills system [21]. These wormholes have a static structure of a "magnetic" gauge field configuration as the $S U(2)$ Yang-Mills wormhole offered in Ref. [16]. On the other hand, in the same $S U(2)$ case, the solution to Yang-Mills equations which exhibit periodic "motion" in Euclidean time was obtained by the author of Ref. [21] recently. Thus we expect the existence of the wormhole solutions which involve two more dynamical variables, in the case where the gauge system has a higher symmetry than $S U(2)$.

In this paper we construct wormhole solutions in the viewpoint of dimensional reduction of gauge fields. Non-Abelian gauge fields can be symmetrically reduced to effective self-interacting scalar fields by the method of coset-space dimensional reduction $[22,23,24]$. We consider a $(1+d)$-dimensional spacetime. In our case, the whole "space," namely, $S^{d}$, is the coset space. We will try to make use of the effective scalar fields to construct the wormhole solutions similar to scalar wormholes. We shall not discuss the stability of the wormhole solutions. Note, however, that classically unstable solutions can largely contribute to the path integral in some special circumstances.

This paper is organized as follows. In Sec. II, we briefly review the cosetspace reduction of Yang-Mills fields, which is necessary to construct wormhole solutions. We then consider first the higher-dimensional cases (Sec. III). In Sec. IV we investigate the wormholes in $1+3$ dimensions. The form of the solution is very different from the solution already known. In Sec. V we present the wormhole solution in $1+2$ dimensions. The Chern-Simons term in the

action is essential to construct the solution. Finally, Sec. VI is devoted to the conclusion.

\section{REDUCTION OF GAUGE FIELDS}

As is well known, a non-Abelian gauge theory symmetric with respect to the canonical action of $G$ on the manifold $M \times G / H$ can be reduced to a gauge theory in $\mathrm{M}$ that includes scalar fields coupled to the gauge field [22, 23, 24]. 
Many people have studied the reduction schemes of the Yang-Mills field in the context of Kaluza-Klein compactification[25]. The scalar potential in the large number of reduction schemes is the Higgs-type potential.

To apply the technique to our system, we use the $d$-dimensional space as a coset space. Now we take the ansatz for the structure of Euclidean space-time. We assume that the wormhole solution has spherical symmetry. The metric can be written as $d s^{2}=d r^{2}+a^{2}(r) d \Omega^{2}\left(S^{d}\right)$, where $d \Omega^{2}\left(S^{d}\right)$ is the line element of the $d$-sphere with unit radius. We consider the symmetric reduction of gauge fields with symmetry group $K$.

The sphere $S^{d}$ is realized in the form of the symmetric spaces $S O(d+$ 1) $/ S O(d)$. If the isotropy group $H$ is simple such as $S O(d)$, then for the simplest embeddings and $\operatorname{rank} G \leq \operatorname{rank} K$ we always have $H \subseteq G \subseteq K$, and vice versa. Moreover, if the embedding $H \subseteq K$ exists for which the homomorphism $\{\tau: H \rightarrow K\}$ can be extended to a homomorphism of $G$ to $K$, the minimum value of the effective scalar potential is zero [23]. Thus in our case, for a sufficiently large gauge group $K \supseteq S O(d+1)$, the minimum value of the potential is zero.

In a typical case, the potential of the reduced theory is of the form [23, 24]

$$
V(\Phi) \propto\left(|\Phi|^{2}-\lambda^{2}\right)^{2},
$$

where the complex variable $\Phi$ comes from the mapping $S^{d}$ to the gauge group $K$. $\Phi(\tau)$ behaves as a scalar field. The potential reaches the minimum value $V=0$ at $|\Phi|^{2}=\lambda^{2}$. Here $\Phi$ has several complex components in general.

The potential comes from $\operatorname{Tr} F_{i j} F^{i j}(i, j=1, \ldots, d)$ in the original YangMills theory. On the other hand, the kinetic term for the effective scalar comes from $\operatorname{Tr} F_{0 i} F^{0 i}$.

The potential depends on $\Phi$ only through $|\Phi|$. Then we obtain a "winebottle"-type potential, and the minima of the potential are mutually connected by the gauge rotation that generates the bottom of this potential.

If we apply the reduction scheme to the construction of the wormhole solutions, we have only to consider the simplest situation. The authors of Ref. [15] showed that the field equations in non-Abelian scalar systems are reduced to be essentially the same as the equations in the $U(1)$-symmetric case described in Refs. $[9,10,11,12,13]$. Therefore, we will consider here $U(1)$-symmetric potential of the wine-bottle type, even if the reduced theory has more symmetries; in that case we simply set the effective field to zero except one complex effective scalar. This "ansatz" is consistent with the field equations in the case with the effective potential of the type (1). We shall restrict ourselves to this class of the effective potential only.

In general, we can rescale the effective scalar field and express the reduced Yang-Mills term as

$$
\operatorname{Tr} F^{M N} F_{M N}=4 d \alpha|\dot{\Phi}|^{2} / a^{2}+2 d(d-1) \beta\left(|\Phi|^{2}-1\right)^{2} / a^{4},
$$

where the numerical coefficients $\alpha$ and $\beta$ are expected to be $\sim 1$, and $M$ and $N$ run over $0,1, \ldots, d$. The complex variable $\Phi$ plays a role of a scalar field. 
Here we set the zeroth component of the gauge field to zero. This is physically justified by a gauge choice. Note that the reduced Lagrangian for the effective scalars depends on the radius of the sphere, $a$.

An example of the representation of $S U(N)$ gauge fields on $S^{d}$ is shown in the Appendix. Since the mapping is not always expressed in so simple a form for arbitrary $d$ and $N$, we show the simple cases where $N \geq d+1$.

In the next section we will apply the reduction (2) to the dynamics of Einstein-Yang-Mills system, and try to find solutions of the wormhole type.

\section{HIGHER-DIMENSIONAL CASES}

We start from a coupled system of gravity and gauge fields in the $(1+d)$ dimensional space-time. In this section we consider the cases with $d \geq 4$. The action is

$$
S=\int d^{d+1} x \sqrt{-g}\left[-\frac{1}{2 \kappa^{2}} R+\frac{1}{4 e^{2}} \operatorname{Tr} F^{M N} F_{M N}\right]+(\text { surface terms }),
$$

where $R$ is the scalar curvature and $F_{M N}$ is the field strength of the non-Abelian gauge fields. The gauge symmetry is assumed to be large enough to have an $S O(d+1)$ group as a subgroup.

The field equations can be derived from the above action. If we assume the metric $d s^{2}=d \tau^{2}+a^{2}(\tau) d \Omega^{2}\left(S^{d}\right)$, and adopt the symmetric reduction of the form (2), the equations for $\Phi$ and $a$ are

$$
\begin{aligned}
& \ddot{\Phi}+(d-2) \frac{\dot{a}}{a} \dot{\Phi}=\frac{d-1}{a^{2}} \frac{\beta}{\alpha}\left(|\Phi|^{2}-1\right) \Phi, \\
& \dot{a}^{2}=1+\frac{1}{d-1} \frac{\kappa^{2}}{e^{2}}\left[2 \alpha \frac{|\dot{\Phi}|^{2}}{a^{2}}-(d-1) \beta \frac{\left(|\Phi|^{2}-1\right)^{2}}{a^{4}}\right],
\end{aligned}
$$

where an overdot denotes a derivative with respect to the Euclidean time $\tau$. Equations (4) and (5) have been derived from the Yang-Mills equation and the time-time component of the Einstein equations.

When we write the complex scalar as $\Phi=f e^{i \psi}, \psi$ is a cyclic variable in terms of the classical mechanics. The equation for $\psi$ is integrable, and this variable is associated with a conserved quantity.

Since we treat "effective" scalar systems and we are interested in the construction of the solution in this paper, we would like to sidestep the complete discussion on the conserved charges and Euclidean formulation. Avoiding the complicated issue about the treatment of conserved charges, we take a conventional view $[11,13,14]$ of the integrable equation.

Using the variables $f$ and $\psi$, one can read the following equation from the imaginary part of the equation of motion for $\Phi$ :

$$
\left(a^{d-2} f^{2} \dot{\psi}\right)^{\cdot}=0 \text {. }
$$


After "Euclideanization", the normalized vector directed in the time coordinate is $i \partial / \partial \tau$; so the normalized derivative of the effective scalar field at the throat takes the real value $[11,13,14]$. Thus we set

$$
a^{d-2} f^{2} \dot{\psi}=i n
$$

where $n$ is a real integration constant. Note that the "charge" $n$ is not the electric or magnetic charge. In our case, the integration constant corresponds to an "external" electric field. Thus we may expect particle creation and quantum instability. We do not consider this an interesting subject here, but we will report on it elsewhere.

Substituting new variables $f$ and (7), the equations of motion (4) and (5) become

$$
\begin{aligned}
& \ddot{f}+(d-2) \frac{\dot{a}}{a} \dot{f}=\frac{\beta(d-1)}{\alpha a^{2}} f\left(f^{2}-1\right)-\frac{n^{2}}{f^{3} a^{2 d-4}} \\
& \dot{a}^{2}=1-\frac{\kappa^{2}}{e^{2}(d-1)}\left[\frac{(d-1) \beta}{a^{2}}\left(f^{2}-1\right)^{2}+\frac{2 \alpha n^{2}}{f^{2} a^{2 d-4}}-2 \alpha \dot{f}^{2}\right] .
\end{aligned}
$$

The "centrifugal" potential term which is proportional to the square of the constant "charge" or "angular momentum" holds the throat of the wormhole so it does not shrink to a zero radius. The sign of the term relative to the other terms is crucial for the existence of the wormhole solutions.

Another important observation is the competition of the centrifugal term and the original potential term. For instance, the first term on the right-hand side of (8) is proportional to $a^{-2}$, while the second term is proportional to $a^{-(2 d-4)}$. The behavior of the wormhole geometry shows that the scale factor a approaches infinity when $\tau$ goes to infinity while the scale factor reaches a finite value when $\tau=0$. If $d \geq 4$, the (attractive) "centrifugal" force dominates around $\tau \simeq 0$ and the potential force dominates at infinity. This feature is analogous to that of the usual scalar wormholes [10]. On the other hand, if $d \leq 3, f$ and $a$ show quite different types of behaviors. These subjects will be investigated in Secs. V and VI.

The rescalings

$$
\tau=K X \quad \text { with } \quad a=K A \quad \text { with } K=\frac{1}{d-1} \frac{\kappa}{e} \frac{\alpha}{\sqrt{\beta}}
$$

lead to the field equations

$$
\begin{aligned}
& f^{\prime \prime}+(d-2) \frac{A^{\prime}}{A} f^{\prime}=\frac{1}{L^{2} A^{2}} f\left(f^{2}-1\right)-\frac{2 q L^{2}}{f^{3} A^{2 d-4}} \\
& \dot{A}^{2}=1-\left[\frac{1}{4 L^{4} A^{2}}\left(f^{2}-1\right)^{2}+\frac{q}{f^{2} A^{2 d-4}}-\frac{f^{\prime 2}}{2 L^{2} A^{2}}\right],
\end{aligned}
$$

with a prime denoting an $X$ derivative and

$$
q=\frac{(d-1) \beta n^{2}}{2 \alpha K^{2 d-6}}, \quad L^{2}=\frac{\alpha}{(d-1) \beta} .
$$


The equations are very similar to those in Ref. [10] except for the dependence of the terms on the scale factor $A$.

We have solved these coupled equations by using the computer code named COLSYS [27]. The resulting solutions are shown in Figs. 1(a) and 1(b) for $d=4$, Figs. 2(a) and 2(b) for $d=5$, and Figs. 3(a) and 3(b) for $d=9$. All the calculations in this section have been performed in the case with $\alpha=\beta=1$.

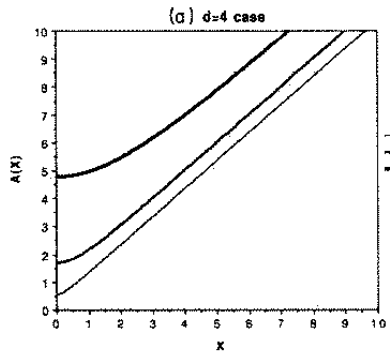

(a)

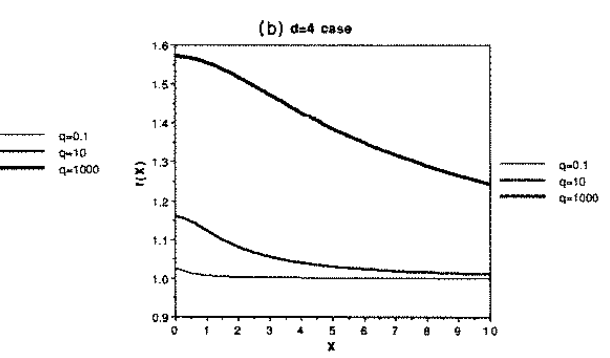

(b)

Figure 1: (a) $A(X)$ as a function of $X$ in the case where the space dimension is four. The lines correspond to the parameter $q=1000,10$, and 0.1 in order of boldness. (b) $f(X)$ as a function of $X$ in the case where the space dimension is four. The definitions of lines are the same as in (a).
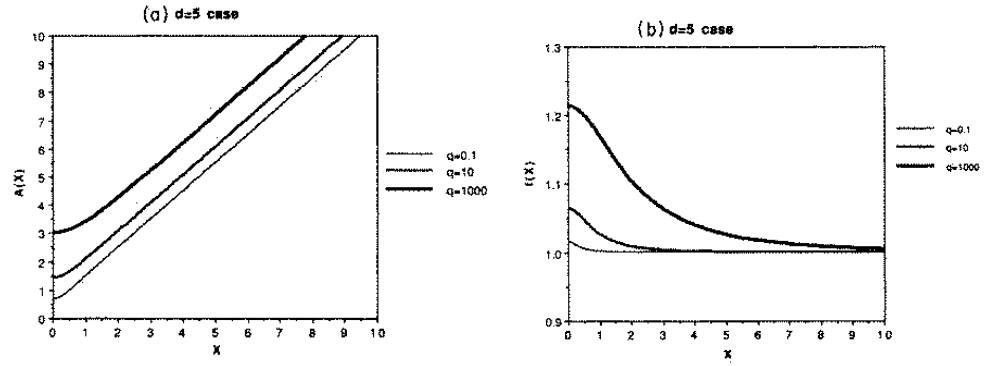

(a) $A(X)$ as a function of $X$ in the case where the space dimension is five. The lines correspond to the parameter $q=1000,10$, and 0.1 in order of boldness.

(b) $f(X)$ as a function of $X$ in the case where the space dimension is five. The definitions of lines are the same as in (a).

Far from the wormhole, i.e., $X \gg 1$, we can ignore gravity and take the Euclidean space-time to be flat, i.e., $A(X) \simeq X$ (Ref. [10]). But we cannot ignore the effective potential for the scalar $f$ completely. Here $f$ goes to one, obeying the effective field equation

$$
f^{\prime \prime}+\frac{d-2}{X} f^{\prime}=\frac{1}{L^{2} X^{2}} f\left(f^{2}-1\right)-\frac{2 q L^{2}}{f^{3} X^{2 d-4}} .
$$



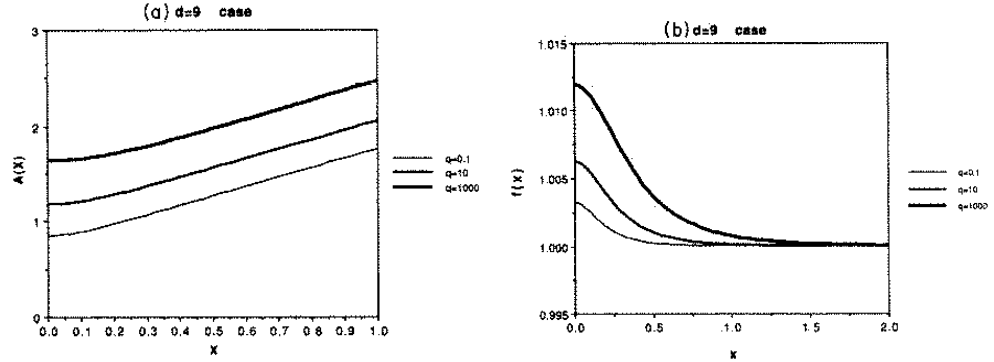

(a) $A(X)$ as a function of $X$ in the case where the space dimension is nine.

The lines correspond to the parameter $q=1000,10$, and 0.1 in order of boldness. (b) $f(X)$ as a function of $X$ in the case where the space dimension is nine. The definitions of lines are the same as in (a).

For large $X$ the first and last terms on the right-hand side dominate and $f$ is obtained up to the leading order

$$
f-1 \simeq X^{-\delta} \quad \text { with } \quad \delta=\frac{d-3+\sqrt{(d-3)^{2}+8 / L^{2}}}{2} .
$$

$f$ in our solutions approaches one according to the negative power of $X$ at large $X$. It can be contrasted with the case for the usual scalar wormhole [10]; in the scalar model with a negative mass term, the value of the scalar field will decrease exponentially to the stationary value. The origin of the difference of the behavior at large $X$ is due to the $A$ dependence of our effective potential.

The case with $d=4$ and $\alpha / \beta=1$ is exceptional, because of the dominance of the second term on the right-hand side in (14). In this case $\delta=2$. In the other cases where we have performed the numerical calculation, (14) is always valid and it gives $\delta=4$ for $d=5$ and $\delta=8$ for $d=9$.

Although $f$ goes to 1 only as the power of $X$, its contribution to the action does not diverge. This is because the effective kinematic term and the potential of the scalar $f$ depends on $A$ in negative power.

Numerical results for $A(0)$ and $f(0)$ as functions of $q$ are shown in Figs. 4(a) and 4(b). We must notice the dependence on $A$ in (11) and (12). Owing to the difference from Eqs. (1.5) and (1.6) in Ref. [10], $f(0)$ increases, rather than decreases, along with the increase of $q$.

These numerical results indicate that the approximation $f \sim 1$ goes well in the range of $q$, in which we have performed the computation $(q<1000)$. This means that we can take

$$
A(0) \sim q^{1 /(2 d-4)} \quad(q<1000) .
$$

For huge values of $q \gg 1000$, it is expected that $A(0) \simeq q^{1 /(2 d-3)}$ and $f(0) \propto q^{2 /(2 d-3)}$, from the analysis of the condition derived from (11) and (12). 


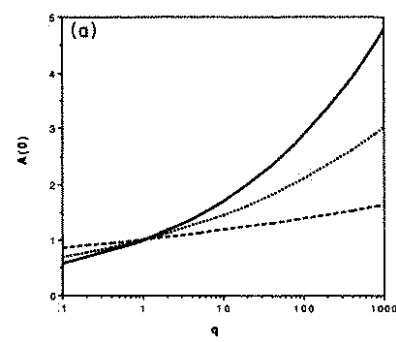

(a)

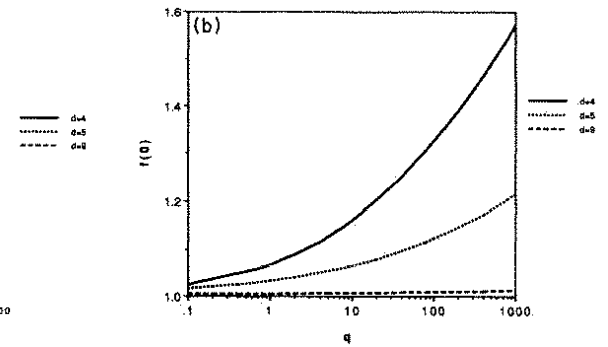

(b)

Figure 2: (a) $A(0)$ as a function of $q$. The solid, dotted, and dashed lines correspond to the space dimension $d=4,5$, and 9 , respectively. (b) $f(0)$ as a function of $q$. The definitions of lines are the same as in (a).

$A(0)$ is related to the size of the wormhole "throat" and also related to the amount of the action. The action of the wormhole solution is of order

$$
S \sim[A(0)]^{d-1} \propto q^{(d-1) /(2 d-4)} \quad(q<1000),
$$

and for a huge value of $q, S \sim q^{(d-1) /(2 d-3)}$.

In Sec. IV the solutions of Eqs. (11) and (12) with $d=3$ will be examined.

\section{THE FOUR-DIMENSIONAL CASE}

In this section we consider the $d=3$ case, i.e., usual four-dimensional spacetime. In Eqs. (11) and (12) in the preceding section, if we set $d=3$, both the "centrifugal" term and the potential term have the same dependence on the scale factor $A$. Moreover, it is possible to factorize the power of $A$ and the equation of the motion for $f$ turns out to be integrable. As a consequence we obtain

$$
\left(A f^{\prime}\right)^{\prime}=\frac{1}{2 L^{2}}\left(f^{2}-1\right)^{2}+\frac{2 q L^{2}}{f^{2}}-2 L^{2} E,
$$

where $E$ is an integration constant and $L^{2}=\alpha /(2 \beta)$. Using this, we can simplify the equation for $A$ :

$$
A^{\prime 2}=1-\frac{E}{A^{2}} .
$$

The size of the wormhole throat is given by $E^{1 / 2}$.

We can solve the equation for $f(X)$ by changing to "conformal (Euclidean) time" $d Y=d X / A$. We get the solution in the form of integration:

$$
Y_{2}-Y_{1}=\int_{f\left(Y_{1}\right)}^{f\left(Y_{2}\right)} \frac{d f}{\sqrt{\left(f^{2}-1\right)^{2} /\left(2 L^{2}\right)+2 q L^{2} / f^{2}-2 E L^{2}}} .
$$


The shape or behavior of the solutions for $f(Y)$ in quality can be revealed by inspection of the equation of motion. We have some different solutions.

If $E<E_{0}$, where $E_{0}$ is defined as the equation

$$
\left(f^{2}-1\right)^{2} /\left(2 L^{2}\right)+2 q L^{2} / f^{2}-2 E_{0} L^{2}=0,
$$

which has a double root, $f(Y)$ increases monotonically from zero to infinity, or shows the time-reversal behavior, in a finite-time interval.

If $E>E_{0}$, there are two types of solutions classified by the region in which $\mathrm{f}$ moves. In one of the cases, $f(Y)$ decreases monotonically from infinity, reaches its minimum value, and increases to infinity again. In the other case, $f(Y)$ increases monotonically from zero, reaches its maximum, and decreases to zero again.

In the case of $E=E_{0}$, there is a solution with constant $f$. However, this solution is "unstable" in the sense of classical dynamics.

All the types of solutions, except for the case of $E=E_{0}$, are singular since $f$ becomes infinite or zero within a finite Euclidean time. In other words, these solutions are singular because $\operatorname{Tr} F^{2}$ diverges. The consideration of a manywormhole configuration, the introduction of Higgs scalars, and the effect of the terms of higher order in $\operatorname{Tr} F^{2}$ in the action are expected to cure the singular behavior and bring about "bounces" at finite values of the field.

Further investigation will be reported in separate publications. Here, we shall estimate the interval between two wormholes or the cutoff scale for a onewormhole configuration.

We restrict ourselves on investigating the case with $E<E_{0}$ and $f<1$. The reader will easily perform a similar analysis in the other case.

The time interval $Y_{i}$ in which $f$ moves from zero to the maximum value is obtained by performing the integration of (20):

$$
Y_{i}=\frac{\sqrt{2} L}{\sqrt{a-c}} F\left(\arcsin \left(\frac{b(a-c)}{a(b-c)}\right)^{1 / 2},\left(\frac{b-c}{a-c}\right)^{1 / 2}\right),
$$

where $F(\phi, k)$ is the elliptic integral of the first kind [28], which is defined as

$$
F(\phi, k)=\int_{0}^{\phi} \frac{d \theta}{\sqrt{1-k^{2} \sin ^{2} \theta}} .
$$

In (21), $a, b, c(a>b>c)$ are the roots of the equation of the third order, $z^{3}-2 z^{2}-\left(4 E L^{4}-1\right) z+4 q L^{4}=0$. The cutoff is required to be less than $Y_{i}$, in order to encounter the singularity, $f \rightarrow 0$.

In an extreme case, $E \gg q \gg, Y_{i} \simeq(q / 2)^{1 / 2} / E L$, and the solution is approximately expressed as

$$
f^{2} \simeq\left(q-8 L^{2} E Y^{2}\right) / 2 .
$$

Since the size of the wormhole throat is $E^{1 / 2}$, the spacetime manifold bounded by the cutoff looks like a "ring of ribbon." Therefore it is doubtful that the usual wormhole dynamics $[2,4,5]$ is applicable in this case. 
On the other hand, if $E$ approaches $E_{0}, Y_{i}$ grows logarithmically. When $q$ is much smaller than one, we get

$$
Y_{i} \simeq \frac{L}{2 \sqrt{2}} \ln \frac{1}{4\left(E-E_{0}\right) L^{4}},
$$

with $E_{0} \simeq q$, whereas $q$ is much larger than one, we have

$$
Y_{i} \simeq \frac{1}{2 \sqrt{2}\left(12 E_{0}\right)^{1 / 4}} \ln \frac{3 E_{0}}{E-E_{0}},
$$

with $E_{0} \simeq \frac{3}{4}\left(2 q L^{4}\right)^{2 / 3} / L^{4}$. In these cases we can take a large cutoff scale.

We can introduce the cosmological constant instead using the cutoff. Then the equation for $A$ is modified as

$$
{A^{\prime}}^{2}=1-\frac{E}{A^{2}}-\frac{\Lambda}{3} A^{2},
$$

where $\Lambda$ is the cosmological constant. We consider that a wormhole attaches to a de Sitter universe of which the radius is approximately $\simeq(3 / \Lambda)^{1 / 2}$. Periodic wormhole solutions with the cosmological constants are considered by authors of Refs. [14] and [18]. One period is likely to be less than or equal to $Y_{i}$, the period of the matter configuration.

We assume that the effect of a higher-derivative term or other effects work in order to avoid the singular behavior inside the de Sitter universe. The present solutions may be cut at about the maximum volume and sewn to the solution of the equation in which higher-derivative terms or other dynamics dominate.

We estimate the interval between the universes. The scale factor $A$ grows from minimum to maximum value in the conformal time interval $Y_{u}$. When $A$ is small, we have $[16,17]$

$$
Y_{u} \simeq \ln \frac{\sqrt{3}}{4 \sqrt{\Lambda E}} .
$$

Therefore the plausible size of the cosmological constants is

$$
\Lambda>\frac{3}{16 E}\left[\frac{1}{4\left(E-E_{0}\right) L^{4}}\right]^{L / 2 \sqrt{2}},
$$

with $E_{0} \simeq q$, whereas $q$ is much larger than one, we have

$$
\Lambda>\frac{3}{16 E}\left[\frac{3 E_{0}}{E-E_{0}}\right]^{L / 2 \sqrt{2}\left(12 E_{0}\right)^{1 / 4}} .
$$

We have estimated the interval between two wormholes, assuming the existence of nonsingular solutions for the Yang-Mills field when the modification of the dynamics is expected. It is necessary to study multiwormhole effects and/or higher-derivative modifications near the singular behavior of our solution in future work.

In the next section we will turn to the case of $d=2$, i.e., the threedimensional case, and look for nonsingular wormhole solutions. 


\section{THREE-DIMENSIONAL WORMHOLE AND CHERN-SIMONS TERM}

In three space-time dimensions, there is no wormhole solution of the type which we treated in Sec. III in a pure Yang-Mills system. If we add $U(1)$ gauge fields to the action we can obtain the wormhole solutions which are kept from collapse by the magnetic charge $[16,21]$.

Here, we do not require the introduction of other fields. Instead, we consider the action which includes the Chern-Simons term [29] as well as the conventional Yang-Mills term.

We consider $S U(2)$ Yang-Mills coupled to gravity. The action is

$$
\begin{aligned}
S=\int d^{3} x & \left\{\sqrt{-g}\left[-\frac{1}{2 \kappa^{2}} R+\frac{1}{4 e^{2}} \operatorname{Tr} F^{M N} F_{M N}\right]\right. \\
+ & \left.\frac{\mu_{E}}{2} \epsilon^{M N L}\left(A_{M} F_{N L}+\cdots\right)\right\}+ \text { (surface terms), }
\end{aligned}
$$

where the ellipsis denotes the term which consists of a triple product of gauge fields.

Because the Chern-Simons term in three dimensions is topological, i.e., it does not include the metric, the Einstein equation is the same as those of pure Yang-Mills theory. The Yang-Mills equations, however, change their form, and also the integration in terms of the "cyclic" variable is modified. The coefficient $\mu_{E}$ of the Chern-Simons term is pure imaginary if the time direction is already taken as Euclidean.

We again use the spherical ansatz on the metric, i.e.,

$$
d s^{2}=d \tau^{2}+a^{2}(\tau)\left(d \theta^{2}+\sin ^{2} d \phi^{2}\right) .
$$

The symmetric reduction of the $S U(2)$ gauge field on $S^{2}$ produces a $U(1)$ symmetric potential with a wine-bottle (or Mexican-hat) shape. This is because $S^{2}=S O(3) / S O(2)$ and $S U(2) \simeq S O(3)$. Thus this is a "minimal" example for the reduction of gauge symmetry.

To express the mapping explicitly we write the gauge fields as

$$
\begin{aligned}
A_{\theta} & =\frac{1}{2}\left(\begin{array}{cc}
0 & -i \Phi e^{-i \phi} \\
i \Phi^{*} e^{i \phi} & 0
\end{array}\right), \\
A_{\phi} & =-\frac{1}{2}\left(\begin{array}{cc}
0 & \Phi e^{-i \phi} \\
\Phi^{*} e^{i \phi} & 0
\end{array}\right) \sin \theta+\frac{1}{2}\left(\begin{array}{cc}
1-\cos \theta & 0 \\
0 & -(1-\cos \theta)
\end{array}\right)
\end{aligned}
$$

where $\theta$ and $\phi$ are the polar and the azimuthal angles of the sphere. Note that here we use a coordinate basis associated with the metric and not an orthonormal one. This mapping leads to the effective Lagrangian of the gauge field

$$
\begin{aligned}
& \frac{1}{4 e^{2}} \operatorname{Tr} F^{M N} F_{M N}+\frac{\mu_{E}}{2 \sqrt{+g}} \epsilon^{M N L}\left(A_{M} F_{N L}+\cdots\right) \\
& =2 \frac{|\dot{\Phi}|^{2}}{a^{2}}+\frac{\left(|\Phi|^{2}-1\right)^{2}}{a^{4}}+\frac{\mu}{2 a^{2}} i\left(\Phi^{*} \dot{\Phi}-\phi \dot{\Phi}^{*}\right),
\end{aligned}
$$


where $\mu=i \mu_{E}$.

Gauge invariance of the quantum theory, which is defined by the path integral, requires quantization of the coupling constant $\mu$. We do not take this fact into account, since we treat only classical equations.

If we write $\Phi$ as $\Phi=f e^{i \psi}$, then we can integrate the equation on $\psi$ and we obtain

$$
\frac{1}{e^{2}} f^{2} \dot{\psi}=i \mu f^{2}+i n,
$$

where $n$ is an integration constant.

We take a unit basis of length so that $\left(\kappa^{2} / e^{2}\right)|n / \mu|$ equals 1 . Moreover we define the parameter set as $\epsilon=|n / \mu|$ and $m^{2}=\mu^{2} e^{4}$. To emphasize the change of the unit basis we write the scale factor as $A$. The scalar variable is rescaled as $F^{2} \equiv f^{2} / \epsilon$.

The field equations are written by using (35) as

$$
\begin{aligned}
\ddot{A} & =\frac{1}{4 \epsilon A^{3}}\left(\epsilon F^{2}-1\right)^{2}, \\
\ddot{F} & =m^{2} F\left(1-\frac{1}{F^{4}}\right)+\frac{1}{A^{2}} F\left(\epsilon F^{2}-1\right) .
\end{aligned}
$$

We consider the case with $\epsilon<1$ only.

The behavior of the possible solution can be seen from (36) and (37). As a wormhole solution we require that $A$ diverges linearly when $\tau$ goes to infinity. Then the value $F$ is attracted to 1 at large $\tau$. Since the original variable $f$ goes to $\sqrt{\epsilon}$, the gauge symmetry is broken at the asymptotic region.

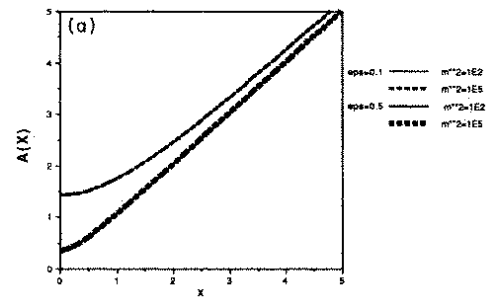

(a)

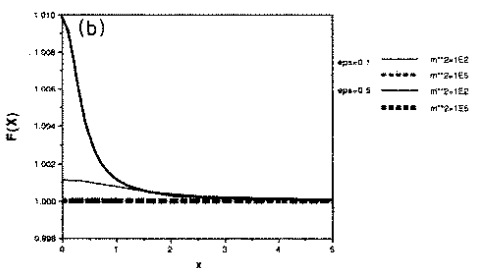

(b)

Figure 3: (a) $A(X)$ as a function of $X$ in the case where the space dimension is two. The solid and dashed lines correspond to the parameter $m^{2}=10^{2}$ and $10^{5}$. At the same time, the thin and thick lines correspond to the parameter $\epsilon=0.1$ and 0.5. (b) $F(X)$ as a function of $X$ in the case where the space dimension is two. The definitions of lines are the same as in (a). The dashed lines overlap one another.

The result of numerical calculations is shown in Figs. 5(a) and 5(b). If e increases, $A(0)$ decreases. On the other hand, $A(0)$ is almost independent of the value of $m^{2}$. No wormhole solution exists if $m$ is less than a certain critical 
value. Because $F(0)$ grows when $\mathrm{m}$ decreases and $F$ cannot grow beyond $1 / \sqrt{\epsilon}$, the critical value exists. Therefore the value depends on $\epsilon$. The details will be published elsewhere.

The action of these wormholes diverges but the divergence is so-called "infrared"; namely, an arbitrary finite cutoff scale (or cosmological constant) makes the action $[10,16,17]$.

The results obtained in this section are rather "academic" ones, but the model we considered is very useful to investigate the connection of matter and gravity in the three-dimensional system, which has attracted much attention recently.

\section{CONCLUSIONS}

We have examined wormhole solutions in non-Abelian gauge theories with a sufficiently large symmetry group. We find that wormhole solutions which are very similar to the scalar wormholes exist in the case where the spatial dimension is larger than three. In these solutions, the gauge symmetry is unbroken in the asymptotic region and the Euclidean action of the solutions is finite. In the case that the spatial dimension equals three, we obtain the solution with the infinite action. The divergence of the action is stronger than the usual divergence [10], because the Lagrangian density of the wormhole solutions itself diverges at finite Euclidean time. This fact suggests that we must consider the "many-body problem" of wormholes seriously in the Yang-Mills system of four dimensional space-time.

The known solutions of Refs. [16] and [17] in the $S U(2)$ Yang-Mills system have an ordinary "infrared divergence" and a finite Lagrangian density. We consider that our model is more generic than those of Refs. [16] and [17]; their model with one effective real scalar is applied to the case with a minimal size of gauge symmetry such as $S U(2)$ on $S^{3}$, while our model with an effective complex scalar can be applied to the case with the arbitrary large gauge group. That is because of the existence of a term such as "centrifugal potential" in the effective theory after the reduction of gauge fields of sufficiently large symmetry.

To avoid the singular behavior we may consider higher-order terms in $F_{M N}$. Note that the finite action can be obtained in the case where the spatial dimension slightly deviates such that $d=4+\epsilon(\epsilon>0)$. Running of the gauge coupling constant may change the behavior of the solutions, and the dilaton coupled to the Yang-Mills fields also affects the behavior of the solutions. These are interesting future problems to study.

\section{ACKNOWLEDGMENTS}

The authors would like to thank A. Nakamula for some useful comments. This work was supported in part by the Grant-in-Aid for Encouragement of Young Scientists from the Ministry of Education, Science and Culture (Grant No. 63790150). 
One of the authors (K.S.) is grateful to the Japan Society for the Promotion of Science for financial support. He also thanks Iwanami Fūjukai for financial aid.

\section{APPENDIX}

In this appendix we will show an example of dimensional reduction of nonAbelian gauge fields on $S^{d}$. Please note that this example is a simple one to express explicit representation, but this reduction may not be a minimal example to obtain a $U(1)$-symmetric effective potential.

We consider the $S U(N)$ Yang-Mills gauge fields in the space of $S^{d}$. Here $N$ must be greater than or equal to $d+1$.

We express the gauge potential as an $N \times N$ matrixvalued one-form A. The generators of $S O(d+1)$ are divided into the generators of $S O(d)$ and those of the complements of $S O(d)$ in $S O(d+1)$. According to this we assume that $\mathbf{A}$ can be decomposed as

$$
\mathbf{A}=\mathbf{A}_{A} e^{A}=\mathbf{A}_{i} e^{i}+\mathbf{A}_{a} e^{a},
$$

where $e^{A}$ are one-forms which satisfy

$$
d e^{A}=\frac{1}{2} f^{A B C} e^{B} \wedge e^{C},
$$

where $f^{A B C}$ is the structure constant of $S O(d+1)$.

Among them, $e^{i}(i=1, \ldots, d)$ transform homogeneously with the $S O(d)$ rotation, i.e.,

$$
d e^{i}=f^{i a j} e^{a} \wedge e^{j} .
$$

On the other hand, $e^{a}, a=1, \ldots, d(d-1) / 2$, obey the Mauer-Cartan equations

$$
d e^{a}=\frac{1}{2} f^{a b c} e^{b} \wedge e^{c}+\frac{1}{2} f^{a i j} e^{i} \wedge e^{j} .
$$

Note that $f^{a b c}$ is the structure constant of the $S O(d)$ subgroup.

The field strength is defined as

$$
F=d \mathbf{A}+\frac{1}{2} i \mathbf{A} \wedge \mathbf{A} .
$$

We require the background configuration of the gauge field. We assume that $\mathbf{A}_{a}$ satisfies

$$
\left[\mathbf{A}_{a}, \mathbf{A}_{b}\right]=i f^{a b c} \mathbf{A}_{c},
$$

where $f^{a b c}$ is the structure constant of $S O(d)$. These configurations can be represented by $\mathrm{d} X \mathrm{X}$ submatrices. Those are just the generators with the appropriate normalization. Further the standard representation of the $S O(d)$ generators in the $d \times d$ matrix form is assumed. Then we set

$$
\left(\mathbf{A}_{i}\right)_{\alpha \beta}=i\left(\delta_{\alpha, d+1} \delta_{\beta, i} \Phi-\delta_{\alpha, i} \delta_{\beta, d+1} \Phi^{*}\right)
$$


where $\alpha$ and $\beta$ denote the component of the matrix. Here we mainly considered the $S O(d+1)$ subgroup of $S U(N)$ and we adopted an additional "phase" to make the complex variable $\Phi(\tau)$.

Taking above "ansatz" with the background geometry $R \times S^{d}$, we obtain

$$
\operatorname{Tr} F_{A B} F^{A B}=4 d|\dot{\Phi}|^{2} / a^{2}+2 d(d-1)\left(1-|\Phi|^{2}\right)^{2} / a^{4},
$$

where $a$ is the radius of $S^{d}$ and the overdot denotes the derivative with respect

to the Euclidean time $\tau$. Equation (45) corresponds to Eq. (2) in the text with $\alpha=\beta=1$.

\section{References}

[1] S. W. Hawking, in 300 Years of Gravitation, edited by S. W. Hawking and W. Israel (Cambridge University Press, Cambridge, England, 1988).

[2] S. Coleman, Nucl. Phys. B310 (1988) 643.

[3] S. Weinberg, Rev. Mod. Phys. 61 (1989) 1.

[4] W. Fischler and L. Susskind, Phys. Lett. B217 (1989) 48.

[5] J. Preskill, Nucl. Phys. B323 (1989) 141.

[6] J. Polchinski, Phys. Lett. B219 (1989) 251.

[7] S. Coleman and K. Lee, Phys. Lett. B221 (1989) 242.

[8] S. Giddings and A. Strominger, Nucl. Phys. B306 (1988) 890.

[9] K. Lee, Phys. Rev. Lett. 61 (1988) 263.

[10] L. F. Abbott and M. B. Wise, Nucl. Phys. B325 (1989) 687.

[11] S. Coleman and K. Lee, Nucl. Phys. B329 (1990) 387.

[12] B. Grinstein, Nucl. Phys. B321 (1989) 439.

[13] J. D. Brown et al., Nucl. Phys. B328 (1989) 213.

[14] S. Midorikawa, Phys. Rev. D41 (1990) 2031.

[15] T. Goto and Y. Okada, Phys. Lett. B237 (1990) 52.

[16] A. Hosoya and W. Ogura, Phys. Lett. B225 (1989) 117.

[17] Y. Verbin and A. Davidson, Phys. Lett. B229 (1989) 364.

[18] R. C. Myers, Phys. Rev. D38 (1988) 1327. 
[19] H. Fukutaka, K. Ghoroku and K. Tanaka, Phys. Lett. B222 (1989) 191;

P. F. Gonzalez-Diaz, ibid. B233 (1989) 85; O. Bertolami, ibid. B234 (1990) 258; S. Hamamoto and Y. Taniguchi, Report No. TOYAMA-64, 1990 (unpublished).

[20] M. B. Green, J. H. Schwarz, and E. Witten, Superstring Theory (Cambridge University Press, Cambridge, England, 1987), Vols. I and II.

[21] A. K. Gupta, J. Hughes, J. Preskill and M. B. Wise, Nucl. Phys. B333 (1990) 195.

[22] N. S. Manton, Nucl. Phys. B158 (1979) 141; P. Forgacs and N. S. Manton, Commun. Math. Phys. 72 (1980) 15; G. Chapline and N. S. Manton, Nucl. Phys. B184 (1981) 391; K. Pilch and A. N. Schellekens, ibid. B256 (1985) 109.

[23] Yu. A. Kobyshin, J. M. Mourao and I. P. Volobujev, Int. J. Mod. Phys. A4 (1989) 151; Nucl. Phys. B322 (1989) 531; I. P. Volobuev, Yu. A. Kubyshin, J. M. Mourao and G. Rudolph, Sov. J. Part. Nucl. 20 (1989) 239.

[24] I. Harnard, S. Shnider and J. Tafel, Lett. Math. Phys. 4 (1980) 107.

[25] T. Appelquist, A. Chodos, and P. G. O. Freund, Modern Kaluza Klein Theories (Benjamin-Cummings, New York, 1987).

[26] J. Schwinger, Phys. Rev. 82 (1951) 664; C. Itzykson and J.-B. Zuber, Quantum Field Theory (McGraw-Hill, New York, 1985), p. 193.

[27] U. Ascher, J. Christiansen, and R. D. Russel, University of British Columbia, Vancouver, Canada, Report No. 77-13, 1977 (unpublished); U. Ascher, J. Christiansen, R. D. Russel, Proceedings of the Conference for Codes for BVP-S in ODE-S, Houston, Texas, 1978 (unpublished).

[28] I. S. Gradshteyn and I. M. Ryzhik, Table of Integrals, Series and Products (Academic, New York, 1965).

[29] S. Deser, R. Jackiw and S. Templeton, Phys. Rev. Lett. 48 (1982) 975; Ann. Phys. (N.Y.) 140 (1982) 372. 